\title{
Bullous pemphigoid induced by Hijama therapy (cupping)
}

\author{
Arghavan Azizpour ${ }^{1}$, Maryam Nasimi $^{1}$, Safoura Shakoei ${ }^{2}$, Fariba Mohammadi $^{1}$, Arsalan Azizpour ${ }^{3}$
}

1 Autoimmune Bullous Diseases Research Center, Tehran University of Medical Sciences (TUMS), Tehran, Iran

2 Department of Dermatology, Imam Khomeini Hospital, Tehran University of Medical Sciences (TUMS), Tehran, Iran

3 Tehran University of Medical Sciences (TUMS), Tehran, Iran

Key words: bullous pemphigoid, Hijama, trigger factor, trauma

Citation: Azizpour A, Nasimi M, Shakoei S, Mohammadi F, Azizpour A. Bullous pemphigoid induced by Hiama therapy (cupping). Dermatol Pract Concept. 2018;8(3):163-165. DOI: https://doi.org/10.5826/dpc.0803a01

Received: November 4, 2017; Accepted: February 11, 2018; Published: July 31, 2018

Copyright: (C2018 Shakoei et al. This is an open-access article distributed under the terms of the Creative Commons Attribution License, which permits unrestricted use, distribution, and reproduction in any medium, provided the original author and source are credited.

Funding: None.

Competing interests: The authors have no conflicts of interest to disclose.

All authors have contributed significantly to this publication.

Corresponding author: Safoura Shakoei, MD, Department of Dermatology, Imam Khomeini Hospital, Tehran University of medical Sciences, Tehran, Iran, 1419733141. Email: dr.shakoei@gmail.com.

ABSTRACT Bullous pemphigoid (BP) is described as a subepidermal blistering disorder, which is commonly reported among the elderly, particularly those older than 60 years of age. In this report, we present the case of a 41-year-old female patient with BP lesions that were initially detected at the site of Hijama therapy with a subsequent generalized spread. Punch biopsy from the lesions and perilesional direct immunofluorescence (DIF) demonstrated features of BP. The anti-BP180 level was $178 \mathrm{RU} / \mathrm{mL}$, and the anti-BP230 level was negative. BP antigens at the site of Hijama caused an antibody response, which led to widespread blistering over the trunk due to epitope spreading. In a comprehensive review of the literature, a total of $22 \mathrm{BP}$ patients with lesions due to physical trauma were studied, and clinical, immunological, and epidemiological information was gathered. This article is the first report on the occurrence of BP induced by Hijama therapy.

\section{Introduction}

Bullous pemphigoid (BP) is a common subepidermal blistering disease. It is a generalized skin disorder that appears as tense bullae on the extremities, flexures, and trunk. Trauma is considered a rare triggering factor for this disease [1]. Hijama therapy (cupping), as mentioned in Islamic Hadiths, is a traditional procedure that employs special cups for pain alleviation. In wet cupping, after the removal of the cups, several small incisions are made at different points of the body and vacuums are used to harvest blood [2]. Herein, we present the case of a female patient (41 years old) with BP lesions at the site of Hijama therapy, which became generalized later on. This case is significant, as this is the first report of BP exacerbated mainly at the site of Hijama therapy.

\section{Case Presentation}

A 41-year-old woman was referred to our hospital with pruritic blisters and erosions at the site of Hijama therapy on her back. A few days earlier, she had undergone cupping without heat on her back that resulted in the local formation of bul- 
lae immediately afterward. The lesions then became generalized, involving the anterior and posterior aspects of the trunk. The patient had similar lesions on her trunk 8 months ago; however, she had not consulted a doctor. Those lesions had spontaneously resolved within 2 months.

The patient's medical and drug histories were insignificant. The physical examination revealed multiple tense bullae and erosions on the trunk, with predominance over the Hijama therapy site (Figure 1). The bullae were about $10-15 \mathrm{~mm}$ in size and filled with a clear fluid; there was no mucosal involvement.

The histopathological examination of the bullae at the Hijama therapy site revealed poor cell subepidermal blistering with a mild perivascular lymphocytic infiltration, as well as eosinophils (Figures 2a-2b). In the basement membrane zone, direct immunofluorescence (DIF) of the perilesional skin revealed the linear C3 deposition (Figure 3). The anti-BP180 level was $178 \mathrm{RU} / \mathrm{mL}$ (positive $>20$ ), and the anti-BP230 level was $7.2 \mathrm{RU} / \mathrm{mL}$ (positive $>20$ ). The anti-desmoglein 1 level was $2 \mathrm{U} / \mathrm{mL}$ (negative $<14$ ), whereas the anti-desmoglein 3 level was $1.7 \mathrm{U} / \mathrm{mL}$ (negative $<14)$. The complete blood cell count, as well as other laboratory findings, was in the normal range.

After reviewing the histopathological and clinical findings at the Hijama therapy site, a diagnosis of BP was confirmed. The patient was started on topical clobetasol propionate $0.05 \%$ (3 tubes per day) that was to be applied on normal skin. Skin lesions improved and no new lesions appeared after 2 weeks. This was followed by tapering of topical corticosteroids over 20 weeks with no signs of relapse.

\section{Discussion}

$\mathrm{BP}$ is described as an autoimmune blistering disease, which involves cellular

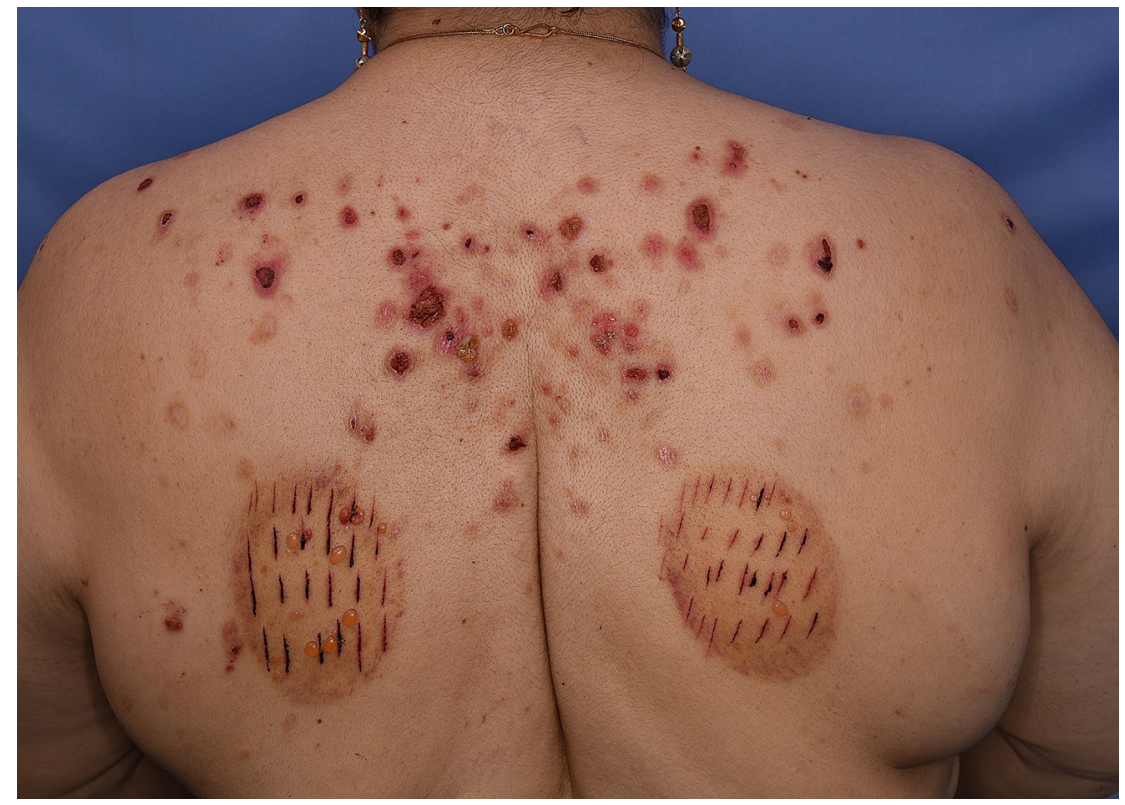

Figure 1. Tense bullae on the trunk, with a preference for the Hijama therapy site. [Copyright: (C2018 Shakoei et al.]

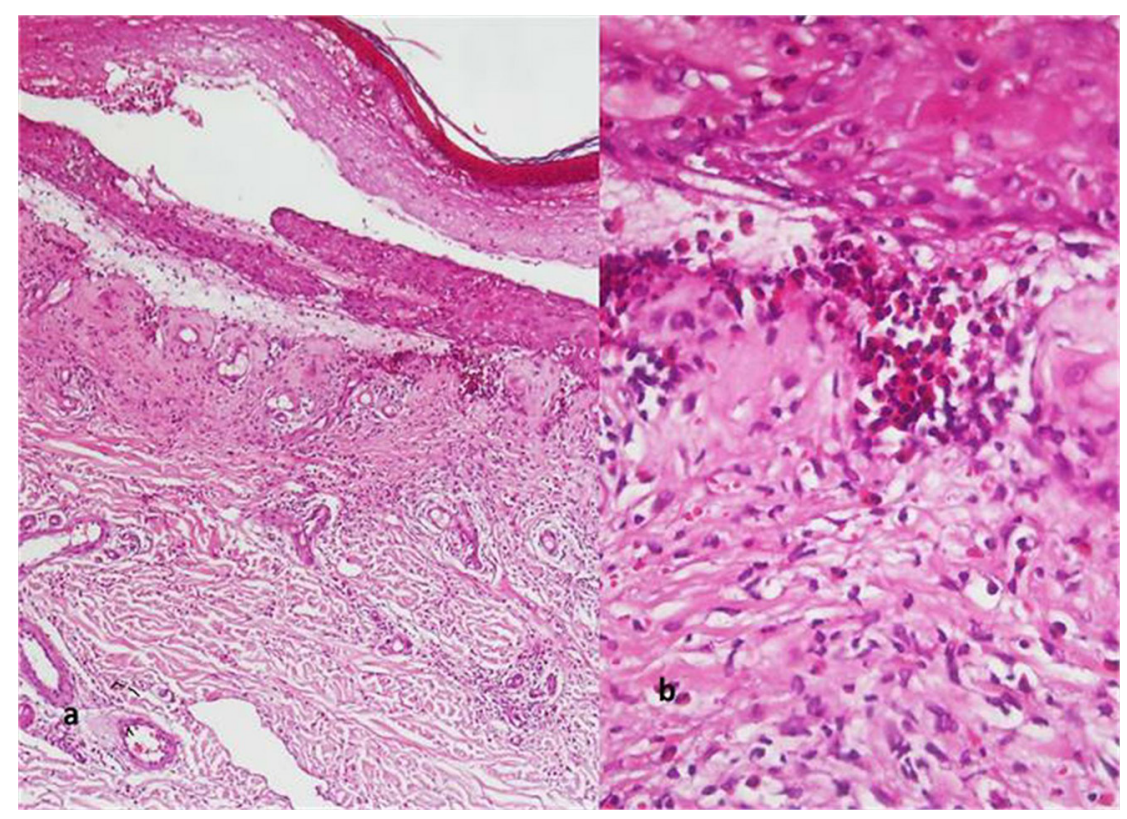

Figure 2. Histopathological features. (a) Subepidermal bulla and eosinophilic infiltrate in the dermis. (b) Severe dermal eosinophilic infiltrate with papillary dermis edema (hematoxylin and eosin stain; magnifications: a×40; b×100). [Copyright: (O2018 Shakoei et al.]

and humoral responses against the cutaneous basement membrane zone. It is commonly reported among the elderly, particularly those older than 60 years of age [1].

Hijama is a complementary therapy used in the treatment of hypertension, polycythemia, headache, migraine, drug intoxication, acne, cough, dyspnea, facial paralysis, lumbar sprain, arthritis, fibromyalgia, soft tissue damage, myofascitis, chronic muscle pain, neurological pain, and chronic nonspecific neck pain $[2,3]$. Various types of cupping include wet cupping, retained cupping, flash cupping, moving cupping, medicinal cupping, and needle cupping.

The Islamic sayings (Hadiths) of Prophet Muhammad emphasize the effectiveness of Hijama therapy for 


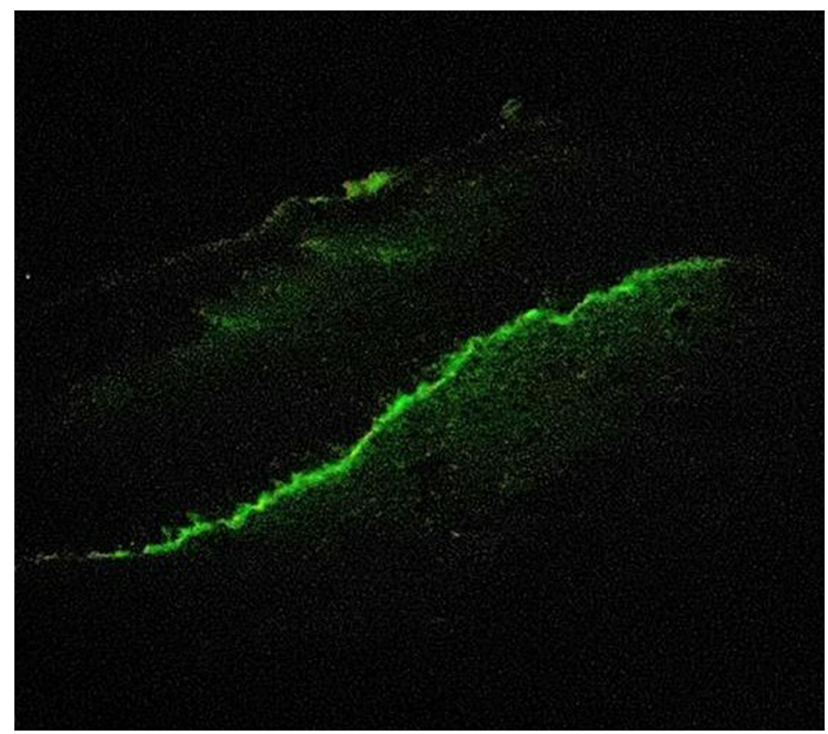

Figure 3. Direct immunofluorescence shows continuous linear deposition of $\mathrm{C} 3$ along the basement membrane zone (magnification ×400). [Copyright: (2018 Shakoei et al.]

health [3]. Due to their religious beliefs, Iranians are interested in Hijama treatment. Many cupping procedures are performed in Iran; however, only $13 \%$ of people consult a doctor prior to Hijama therapy [3]. Wet cupping is performed to ease the pain through local suctioning with special cups on the selected areas, which are often the sites of pain. Following the removal of the cup, a sterile scalpel is used to make small skin incisions. The cup is then placed on the incision site in order to draw blood and remove toxins [2].

Trauma (both sucking and abrasions) can change the vascular permeability and trigger deposition of autoantibodies in patients with subclinical BP [4]. Various factors, including trauma, friction, insect bites, surgical wounds, burns, skin grafting, radiotherapy, photo chemotherapy, and venous access procedures, have been reported to induce lesions [5].

There are several theories for the induction of BP lesions due to trauma. First, disruption of the skin due to trauma might increase the expression of the basement membrane zone antigens, leading to the initiation of an inflammatory process with lower titers of circulating antibodies. Due to the increased storage of circulating autoantibodies, besides inflammatory cell recruitment (particularly granulocytes), tissue damage can improve vascular permeability $[4,6]$. By attaching to granulocytes, autoantibodies stimulate the complement system and initiate a loop of inflammatory stimuli [4]. Proteolytic enzymes of activated granulocytes, such as matrix metallopeptidase-9, degrade the BP180 extracellular domain and can lead to the formation of bullae [4].

Physical factors, including UV radiation, radiotherapy, and irradiation, can alter the basal membrane and expose modified antigens, leading to the stimulation of autoantibody formation, complement activation, and blister formation [6]. UV radiation and/or radiotherapy decrease T-cell immune reactivity and lead to the development of antibodies targeted against the proteins [4].

The epitope spreading phenomenon explains the extension of BP lesions. The targets of immune responses can even be other epitopes of the same protein or proteins from the same tissue [4]. According to the review by Dănescu, the most common factor inducing BP lesions is radiotherapy. The findings showed that the interval between the onset of BP lesions and activity of the trigger factor was less than 1 month, similar to our report [4]. The onset of new lesions may be only limited to the site of trauma, or it may spread to other body parts, as seen in our patient [4].

\section{Conclusion}

This article is the first report on the occurrence of BP lesions induced by Hijama therapy. Despite Hijama therapy-induced BP lesions being an uncommon presentation, physicians should be cautious about the risks and consider the diagnosis of BP in patients with blisters arising from Hijama therapy.

\section{References}

1. Nguyen T, Kwan JM, Ahmed AR. Relationship between radiation therapy and bullous pemphigoid. Dermatology. 2014;229:88-96. doi: $10.1159 / 000362208$.

2. Sajid MI. Hijama therapy (wet cupping)-its potential use to complement British healthcare in practice, understanding, evidence and regulation. Complement Ther Clin Pract. 2016;23: 9-13. doi: 10.1016/j.ctcp.2016.01.003.

3. Vakilinia SR, Bayat D, Asghari M. Hijama (wet cupping or dry cupping) for diabetes treatment. Iran J Med Sci. 2016;41:S37.

4. Dănescu S, Chiorean R, Macovei V, Sitaru C, Baican B. Role of physical factors in the pathogenesis of bullous pemphigoid: case report series and a comprehensive review of the published work. J Dermatol. 2016;43:134-140. doi: 10.1111/1346-8138.13031.

5. Korfitis C, Gregoriou S, Georgaia S, Christofidou E, Danopoulou I. Trauma-induced bullous pemphigoid. Indian J Dermatol Venereol Leprol. 2009;75:617-619. doi: 10.4103/0378-6323.57732.

6. Isohashi F, Konishi K, Umegaki N, et al. A case of bullous pemphigoid exacerbated by irradiation after breast conservative radiotherapy. Jpn J Clin Oncol. 2011; 41:811-813. doi: 10.1093/jjco/ hyr049. 